\title{
Two-dimensional transport and transfer of a single atomic qubit in optical tweezers
}

\author{
JÉRÔME BEUGNON, CHARLES TUCHENDLER, HAROLD MARION, ALPHA GAËTAN, \\ YEVHEN MIROSHNYCHENKO, YVAN R. P. SORTAIS, ANDREW M. LANCE, MATTHEW P. A. JONES, \\ GAÉTAN MESSIN, ANTOINE BROWAEYS* AND PHILIPPE GRANGIER
}

\author{
Laboratoire Charles Fabry de I'Institut d'Optique, CNRS, Univ. Paris-sud, Campus Polytechnique, RD 128, 91127, Palaiseau cedex, France \\ *e-mail: antoine.browaeys@institutoptique.fr
}

Quantum computers have the capability of out-performing their classical counterparts for certain computational problems ${ }^{1}$. Several scalable quantum-computing architectures have been proposed. An attractive architecture is a large set of physically independent qubits arranged in three spatial regions where (1) the initialized qubits are stored in a register, (2) two qubits are brought together to realize a gate and (3) the readout of the qubits is carried out ${ }^{2,3}$. For a neutral-atom-based architecture, a natural way to connect these regions is to use optical tweezers to move qubits within the system. In this letter we demonstrate the coherent transport of a qubit, encoded on an atom trapped in a submicrometre tweezer, over a distance typical of the separation between atoms in an array of optical traps ${ }^{4-6}$. Furthermore, we transfer a qubit between two tweezers, and show that this manipulation also preserves the coherence of the qubit.

In the quest for an implementation of a quantum computer, scalability is a major concern. In the trapped-ion approach (see for example ref. 7), a lot of effort is being devoted to building arrays of small ion traps ${ }^{8}$, and to moving ion qubits whilst avoiding heating and decoherence ${ }^{9}$. Neutral atoms also offer promising properties for the realization of large quantum registers. For example, one- or two-dimensional addressable arrays of dipole traps have been demonstrated using holographic techniques, ${ }^{4}$, micro-fabricated elements ${ }^{5}$, or active rearrangement of single atoms $s^{6,10}$. An alternative approach is to use the Mott insulator transition to initialize a three-dimensional register by loading a Bose-Einstein condensate into an optical lattice ${ }^{11}$. Recent progress has shown subwavelength addressability in such a system ${ }^{12}$. To carry out quantum computations, however, an additional key feature is the ability to realize the gate between two arbitrary qubits of the register.

Here we demonstrate a scheme where a neutral-atom qubit is transferred between two moving tweezers ('register' to 'moving head'), and then transported towards an interaction zone where the two-qubit gate should be implemented ${ }^{13-16}$. We show that these manipulations of the external degrees of freedom preserve the coherence of the qubit, and do not induce any heating. This transport in a moving tweezer is a promising alternative to the recently demonstrated transport of qubits in 'optical conveyor belts ${ }^{6,17}$, or in state-dependent moving optical lattices ${ }^{18}$. Altogether, these results could pave the way towards a scalable neutral-atom quantum-computing architecture.

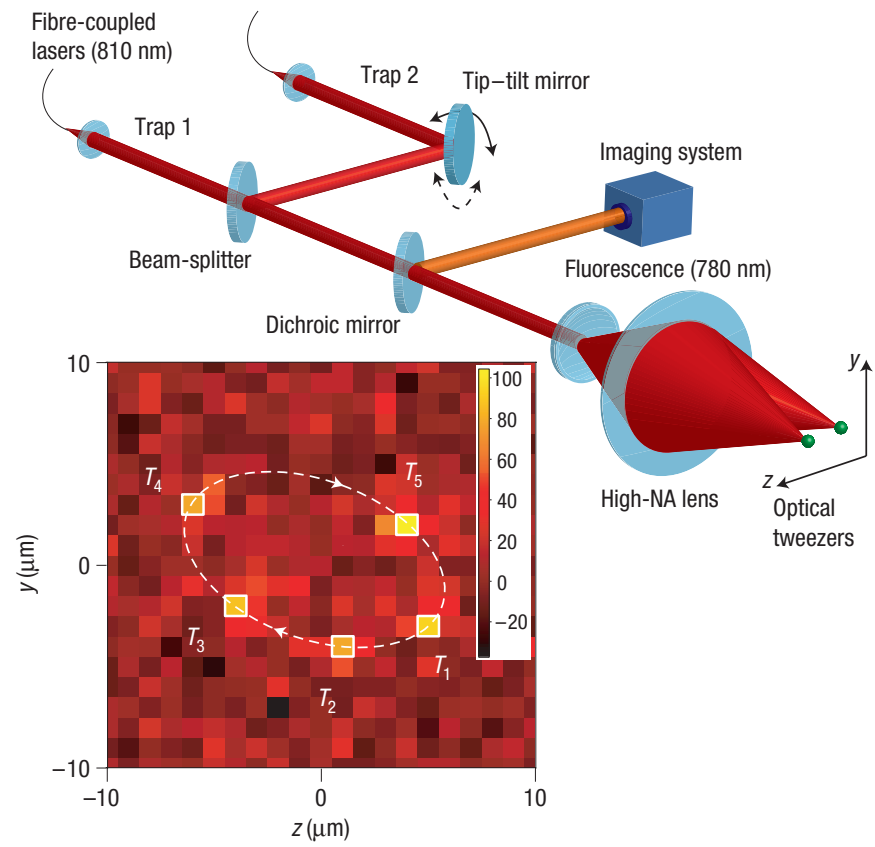

Figure 1 Experimental set-up. A large-numerical-aperture lens focuses two independent dipole-trap beams at $810 \mathrm{~nm}$ each to a size of $0.9 \mu \mathrm{m}$. An optical power of $400 \mu \mathrm{W}$ results in a trap depth of $500 \mu \mathrm{K}$ and oscillation frequencies of 81 and $15 \mathrm{kHz}$, in the radial and axial directions respectively. The two trapping lasers have the same linear polarization and their frequencies are separated by $10 \mathrm{MHz}$ to avoid interference. The moving tweezer is displaced by rotating a tip-tilt platform. The same large-numerical-aperture lens is used to collect the fluorescence light at $780 \mathrm{~nm}$ from the atom. This fluorescence light is separated from the trapping light by the dichroic mirror and sent to a single-photon counter module and a CCD (charge-coupled device) camera. The inset shows a fluorescence picture of an atom moved along an elliptical trajectory in the $y-z$ plane, thus showing the two-dimensional control of the displacement. The picture is a summation of five images taken at different times during the motion. The colour scale represents the number of counts per pixel of the CCD camera (background substracted).

In our experiment, we trap a single rubidium-87 atom in an optical dipole trap created by a tightly focused laser beam ${ }^{19,20}$. 

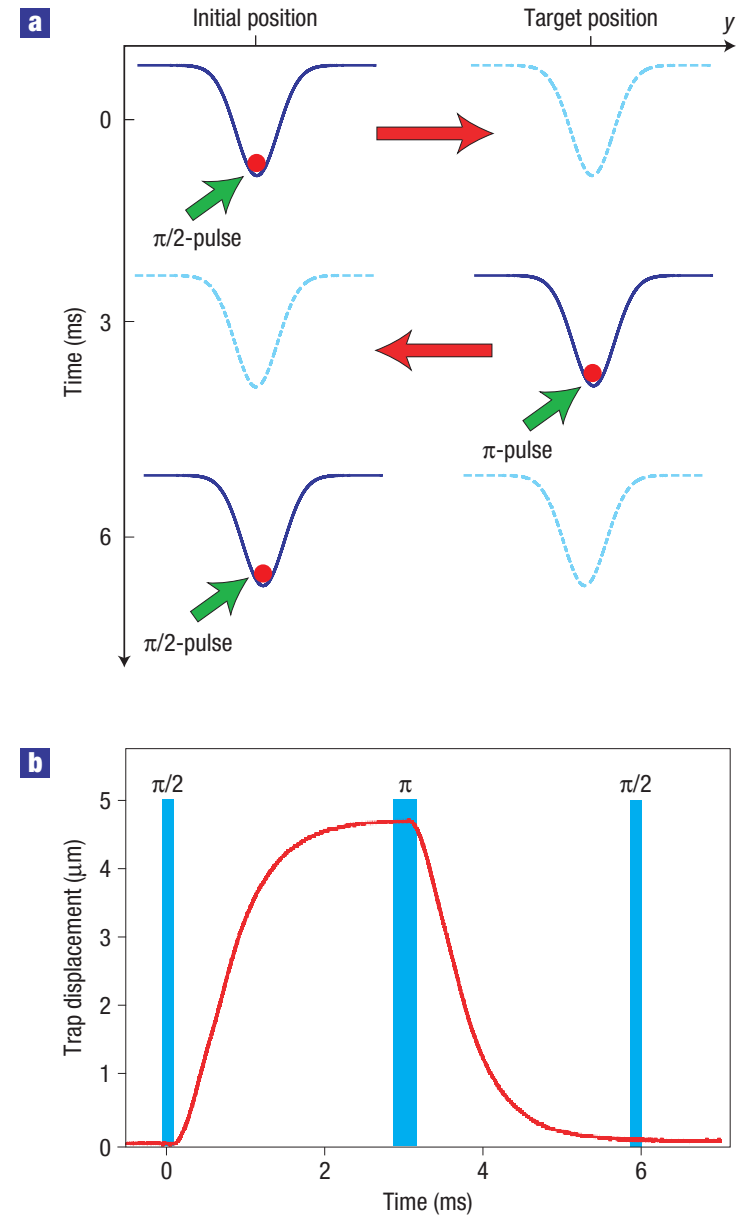

Figure 2 Principle of the moving-qubit experiment. a, Position of the atom when the pulses of the spin-echo sequence are applied. Starting at the initial position, the first pulse prepares the atom in a superposition $(|0\rangle+|1\rangle) / \sqrt{2}$. The tweezer is then moved along the $y$ axis to the target position and the $\pi$-pulse is applied. Finally, the tweezer is brought back to its initial position and the coherence is checked by applying a second $\pi / 2$-pulse and measuring the state of the qubit. $\mathbf{b}$, Example of displacement of the tweezer versus time. The signal is obtained from the sensor attached to the tip-tilt platform, which is converted into a distance travelled by the tweezer. The $\pi / 2$ - and $\pi$-pulses, being 2 and $4 \mu$ s long respectively, are not to scale.

As described in detail in ref. 21, the qubit is encoded onto the $|0\rangle=|F=1, M=0\rangle$ and $|1\rangle=|F=2, M=0\rangle$ hyperfine ground states separated by $\omega_{\mathrm{hf}} \approx 6.8 \mathrm{GHz}$. We initialize the qubit in state $|0\rangle$ by optically pumping the atom. We drive single-qubit operations by a Raman transition using two phased-locked laser beams, one of which is the dipole trap. The internal dephasing time of the qubit, measured by Ramsey interferometry, is approximately $630 \mu \mathrm{s}$. This time is mostly limited by the residual motion of the atom in the trap, which leads to a fluctuation of the frequency of the qubit transition. This dephasing can be reversed by applying a spinecho technique, where a $\pi$-pulse is inserted between the two $\pi / 2$ pulses of the Ramsey sequence. Using this technique we measure an irreversible dephasing time of $34 \mathrm{~ms}$.

The experimental set-up for the moving tweezer is represented in Fig. 1. We reflect the dipole-trap beam off a mirror affixed to a tip-tilt platform before the large-numerical-aperture lens. The platform is actuated by piezo-electrical transducers and can rotate
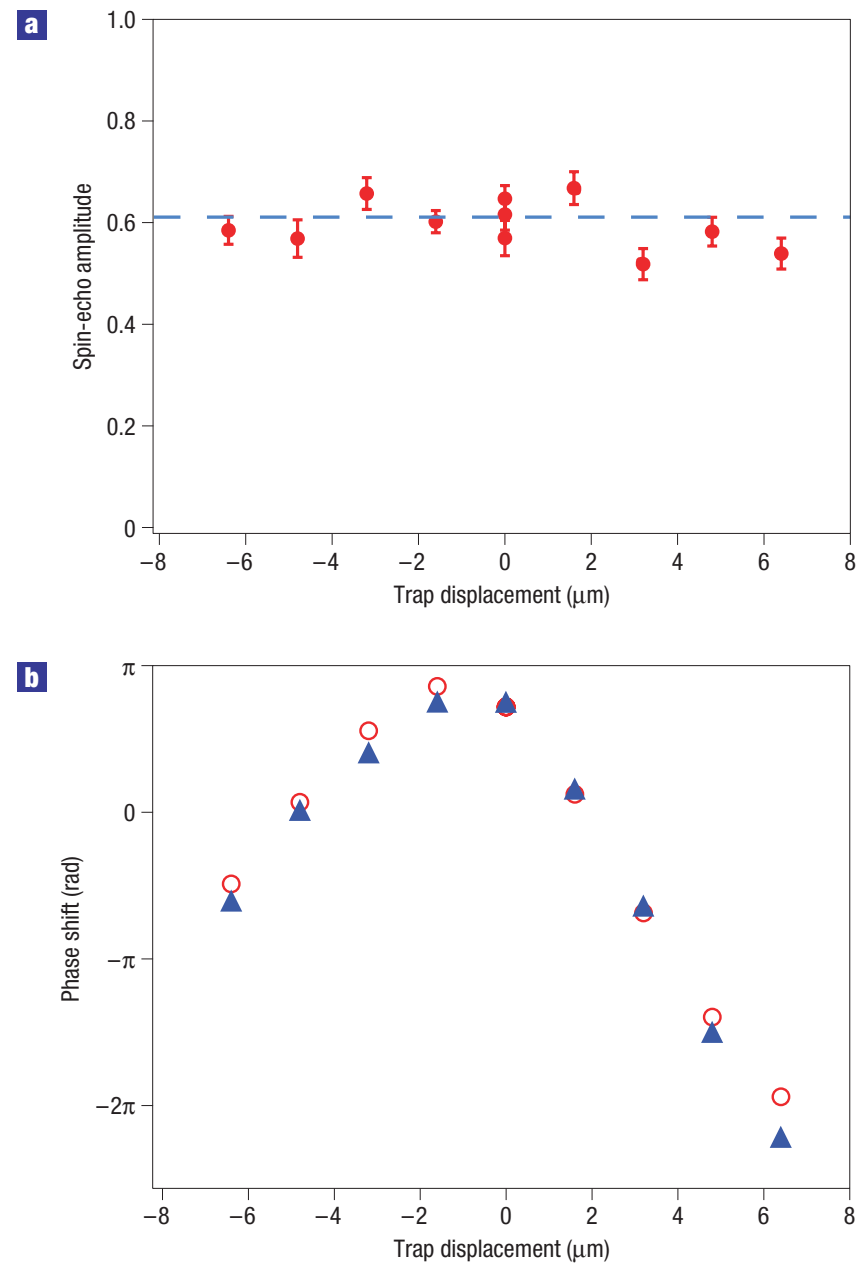

Figure 3 Results of the moving-qubit experiment. a, The amplitude of the spin-echo signal versus the amplitude of the displacement. The error bars are the root-mean-square (r.m.s.) uncertainty obtained from the fit of the fringes. The $60 \%$ contrast is a result of the damping of the fringes after $6 \mathrm{~ms}$ (ref. 21). The dashed line represents the average of the data for no trap displacement. $\mathbf{b}$, The phase shift versus the amplitude of the displacement. The triangles are the data and the circles are the calculated values of the dephasing on the basis of the model described in the Methods section.

with a maximal angle of $2.5 \mathrm{mrad}$ in both the horizontal and vertical directions. We have measured the position of the dipole trap for different angles of the platform by observing the position of the atom on the CCD camera. The maximal angle corresponds to a total displacement of the tweezer of $18 \pm 1 \mu \mathrm{m}$. This motion is two dimensional, as demonstrated in Fig. 1.

We first analyse the influence of a displacement of the tweezer on the external degrees of freedom of the qubit. For this purpose, we measure the temperature of the single atom in the tweezer using a release-and-recapture technique ${ }^{22}$ (see the Methods section). In the absence of motion, the temperature of the atom is $56.0 \pm 1.4 \mu \mathrm{K}$. We repeat this measurement after moving the tweezer by a total distance of $360 \mu \mathrm{m}$, consisting of 20 round trips of $18 \mu \mathrm{m}$, along the $z$ axis. Each round trip lasts a time of $6 \mathrm{~ms}$. We do not find any measurable loss due to this transport. After the motion, we measure a temperature of $54.8 \pm 1.6 \mu \mathrm{K}$. As the energy difference between two vibrational quanta in the radial direction is $4 \mu \mathrm{K}$, this temperature is compatible with no change on average in the 
a
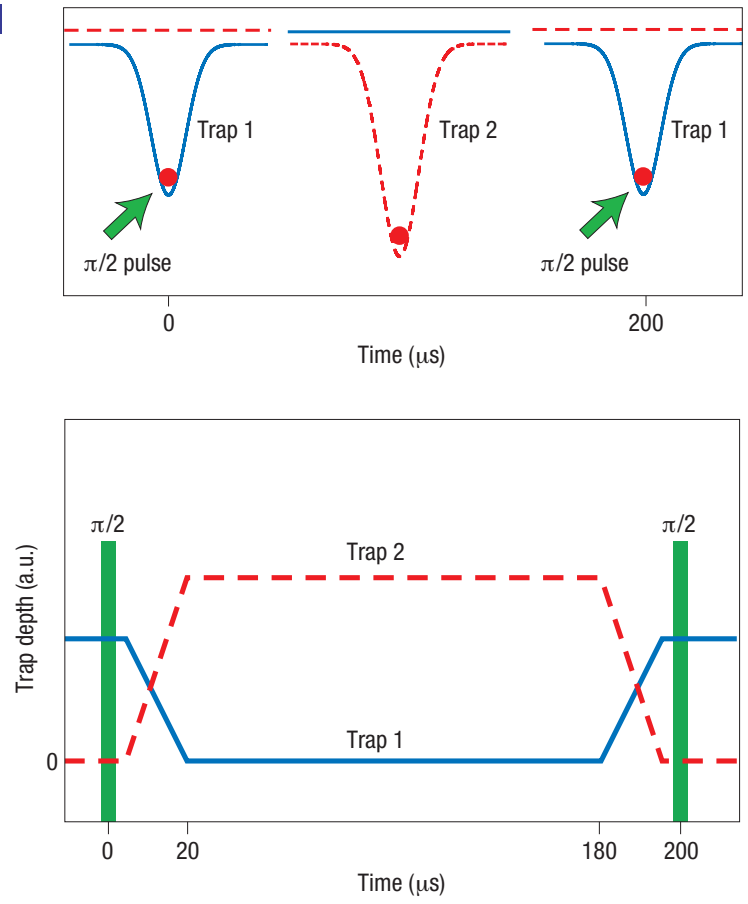

b

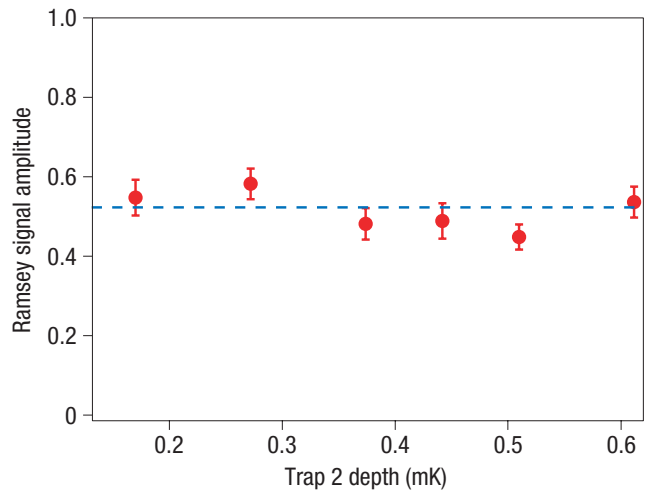

$\boldsymbol{G}$

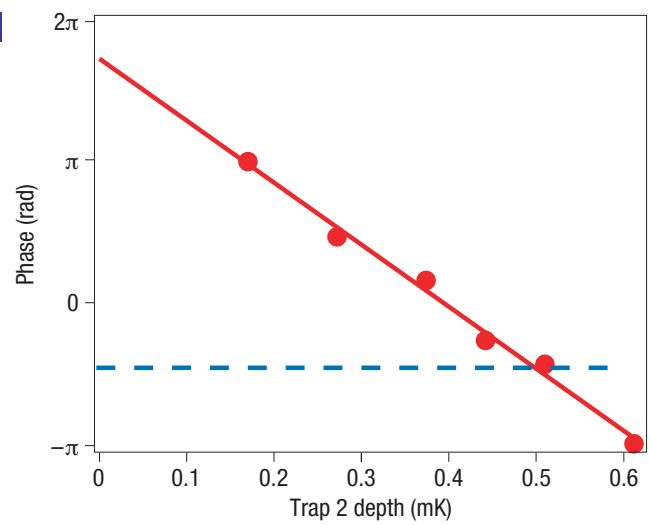

Figure 4 Experiment on the transfer of the qubit between two tweezers. a, Details of the time sequence. $A \pi / 2$-pulse is applied when the atom is initially in trap 1 , which has a depth of $500 \mu \mathrm{K}$. The atom is transferred to trap 2 in a time of $20 \mu \mathrm{s}$. After a time of $160 \mu \mathrm{s}$, the atom is transferred back to trap 1 and a second $\pi / 2$-pulse is applied. $\mathbf{b}$, The amplitude of the Ramsey oscillations as a function of the depth of the trap into which the atom is transferred. The error bars are the r.m.s. uncertainty obtained from the fit of the fringes. c, The phase of the Ramsey oscillations for different depths of the second trap. The solid line is a linear fit to the data. The dashed lines in b and $\mathbf{c}$ correspond to no transfer.

radial vibrational state. This absence of motional heating is a crucial feature for entanglement schemes on the basis of controlled collisions $^{16,23}$ and results from the adiabaticity of the displacement. A motion is adiabatic if the acceleration $a$ fulfils $m a \sigma \ll \hbar \Omega$ ( $\Omega$ is the oscillation frequency of the atom, $m$ its mass and $\sigma$ the extension of the ground-state wavefunction $\left.{ }^{24}\right)$. This gives a maximum acceleration of $\approx 10^{4} \mathrm{~m} \mathrm{~s}^{-2}$, much larger than the experimentally measured $\approx 15 \mathrm{~m} \mathrm{~s}^{-2}$.

Second, we study the influence of the motion on the coherence of the qubit. As the duration of the transport is larger than the dephasing time of the qubit $(630 \mu \mathrm{s})$, we apply the spinecho sequence to rephase the qubit ${ }^{21}$. The time sequence of the experiment is shown in Fig. 2. Figure 3a shows the amplitude of the spin-echo fringes for various trap displacements along the $y$ axis. This amplitude is constant when we scan the tweezer over the whole transverse field of the objective. This demonstrates that the motion does not affect the internal coherence of the qubit. To show the independent two-dimensional control of our system, we move the tweezer along the $z$ axis and observe the same behaviour as along the $y$ axis.

We also observe a phase shift of the spin-echo fringes, as shown in Fig. 3b. This is evidence that the two states of the qubit dephase with respect to each other during the motion (in a reproducible way), despite the presence of the rephasing $\pi$-pulse. We attribute this phase shift to the asymmetry of the trajectory during the first and second parts of the round-trip displacement. We have modelled this effect and found a good agreement with the data (see the
Methods section). This understanding of the phase evolution of the qubit during the motion is crucial for a possible implementation in a quantum computer, where qubit phases need to be controlled.

With the idea of transferring an atom from the 'register' to the 'moving head', we have investigated the transfer of a qubit from one tweezer to a second one. For this experiment, the two traps are superimposed and the positions of both tweezers are fixed. The experimental sequence is shown in Fig. 4a. We load an atom in the first tweezer, transfer it to a second tweezer, and transfer it back to the first tweezer, with no measurable loss. When the two traps have the same depth, we measure a temperature of the atom after the double transfer of $56.3 \pm 1.8 \mu \mathrm{K}$, whereas the temperature with no transfer is $53.4 \pm 1.4 \mu \mathrm{K}$. Therefore, the transfer does not induce any significant motional heating.

We analyse the influence of the transfer on the coherence of the qubit by inserting the double transfer between the two $\pi / 2$-pulses of a Ramsey sequence, as shown in Fig. 4 a. Figure 4 presents the amplitude and the phase of the Ramsey fringes after this sequence for depths of the second tweezer ranging from 0.2 to $0.6 \mathrm{mK}$. This transfer does not affect the amplitude of the Ramsey signal when the depth of the second trap is varied, thus showing that the coherence is robust against the transfer between the two traps.

Figure $4 \mathrm{c}$ shows that the phase of the Ramsey fringes varies linearly with respect to the depth of the second trap. This is explained by the differential potential experienced by the two states, which is proportional to the depth of the trap $U$. If the depths of the two traps differ by $\Delta U$, the Ramsey fringes are shifted after a 
holding time $T$ by a phase proportional to $\Delta U T / \hbar$, with respect to the situation where no transfer is applied. This phase is thus a useful tool to make sure that the two traps are identical.

In conclusion, we have shown that we can move and transfer a single qubit between two tweezers with no measurable motional heating. We have also shown no loss of coherence of the atomic qubit under transfer and displacement. In combination with our holographic array of dipole traps ${ }^{4}$, and efficient single-qubit operation and readout ${ }^{21}$, we have made a first step towards designing a scalable architecture of a quantum computer on the basis of neutral atoms.

\section{METHODS}

\section{TEMPERATURE MEASUREMENT}

After trapping a single atom, we switch off the dipole trap for a time adjustable between 1 and $\sim 30 \mu$ s. We then turn the trap back on and check for the presence of the atom. We repeat this sequence 100 times for each release time and calculate the probability of recapturing the atom after the corresponding time of flight. We compare our data with a three-dimensional Monte Carlo simulation, taking into account the potential produced by the gaussian trapping beam, and assuming a thermal distribution of the position and the velocity of the atom at the beginning of the time of flight. The error bar of this fitted temperature corresponds to one standard deviation in the least-square-based fit.

\section{PHASE SHIFT DURING THE MOTION}

The hyperfine splitting of $6.8 \mathrm{GHz}$ means that the dipole trap detuning is slightly larger for $|0\rangle$ than for $|1\rangle$, giving rise to a small differential light-shift. Therefore, the qubit transition frequency is $\omega_{\mathrm{hf}}+\eta U / \hbar$, with $U$ the depth of the dipole trap and $\eta=7 \times 10^{-4}$ for our trap. If the tweezer is not moved, the $\pi$-pulse compensates for the phase accumulated during the two parts of the motion. When the tweezer is moved off axis, the waist of the beam increases slightly, resulting in a shallower trap. Figure 2a shows that with the tweezer starting on axis the atom spends more time far from the axis, where the dipole trap is shallower, whereas on the way back it spends more time around the axis, where the dipole trap is stronger. The average depths are then different for the two parts of the motion, and so are the phases. As the phase of the spin-echo signal is the difference of the phases accumulated during the two periods of the motion, it is expected to vary as we move the tweezer further away off axis. We have modelled this effect by calculating the dephasing accumulated during the transport, taking into account the actual displacement of the tweezer from the sensor curve and the measured Rabi frequencies for different positions of the tweezer off axis. The result of this model is shown as circles in Fig. $3 \mathrm{~b}$ and is consistent with the data.
Received 27 April 2007; accepted 12 July 2007; published 12 August 2007.

\section{References}

1. Nielsen, M. A. \& Chuang, I. L. Quantum Computation and Quantum Information (Cambridge Univ. Press, Cambridge, 2000)

2. Kielpinski, D., Monroe, C. \& Wineland, D. J. Architecture for a large-scale ion-trap quantum computer. Nature 417, 709-711 (2002).

3. Calarco, T., Dorner, U., Julienne, P. S., Williams, C. J. \& Zoller, P. Quantum computations with atoms in optical lattices: Marker qubits and molecular interactions. Phys. Rev. A 70, 012306 (2004).

4. Bergamini, S. et al. Holographic generation of micro-trap arrays for single atoms using a programmable phase modulator. J. Opt. Soc. Am. B 21, 1889-1894 (2004).

5. Dumke, R. et al. Micro-optical realization of arrays of selectively addressable dipole traps: A scalable configuration for quantum computation with atomic qubits. Phys. Rev. Lett. 89, 097903 (2002).

6. Miroshnychenko, Y. et al. Quantum engineering: An atom-sorting machine. Nature 442, 151 (2006).

7. Chiaverini, J. et al. Implementation of the semiclassical quantum Fourier transform in a scalable system. Science 308, 997-1000 (2005)

8. Seidelin, S. et al. Microfabricated surface-electrode ion trap for scalable quantum information processing. Phys. Rev. Lett. 96, 253003 (2006).

9. Rowe, M. A. et al. Transport of quantum states and separation of ions in a dual RF ion trap. Quantum Inf. Comput. 2, 257-271 (2002).

10. Schrader, D. et al. Neutral atom quantum register. Phys. Rev. Lett. 93, 150501 (2004)

11. Greiner, M., Mandel, O., Esslinger, T., Hänsch, T. W. \& Bloch, I. Quantum phase transition from a superfluid to a Mott insulator in a gas of ultracold atoms. Nature 415, 39-44 (2002).

12. Lee, P. J. et al. Sublattice addressing and spin-dependent motion of atoms in a double-well lattice. Phys. Rev. Lett. 99, 020402 (2007).

13. Jaksch, D., Briegel, H. J., Cirac, J. I., Gardiner, C. W. \& Zoller, P. Entanglement of atoms via cold controlled collisions. Phys. Rev. Lett. 82, 1975-1978 (1999).

14. Brennen, G. K., Caves, C. M., Jessen, P. S. \& Deutsch, I. H. Quantum logic gates in optical lattices. Phys. Rev. Lett. 82, 1060-1063 (1999).

15. Jaksch, D. et al. Fast quantum gates for neutral atoms. Phys. Rev. Lett. 85, 2208-2211 (2000).

16. Dorner, U., Calarco, T., Zoller, P., Browaeys, A. \& Grangier, P. Quantum logic via optimal control in holographic dipole traps. J. Opt. B 7, S341-S346 (2005).

17. Kuhr, S. et al. Coherence properties and quantum state transportation in an optical conveyor belt. Phys. Rev. Lett. 91, 213002 (2003).

18. Mandel, O. et al. Coherent transport of neutral atoms in spin-dependent optical lattice potentials. Phys. Rev. Lett. 91, 010407 (2003).

19. Schlosser, N., Reymond, G. \& Grangier, P. Collisional blockade in microscopic optical dipole traps. Phys. Rev. Lett. 89, 023005 (2002).

20. Sortais, Y. R. P. et al. Diffraction-limited optics for single-atom manipulation. Phys. Rev. A 75, 013406 (2007).

21. Jones, M. P. A. et al. Fast quantum state control of a single trapped neutral atom. Phys. Rev. A 75, 040301(R) (2007).

22. Lett, P. D. et al. Observation of atoms laser cooled below the Doppler limit. Phys. Rev. Lett. 61, 169-172 (1988).

23. Mandel, O. et al. Controlled collisions for multi-particle entanglement of optically trapped atoms. Nature 425, 937-940 (2003).

24. Browaeys, A. et al. Transport of atoms in a quantum conveyor belt. Phys. Rev. A 72, 053605 (2006).

\section{Acknowledgements}

We would like to thank W. D. Phillips, T. Porto, I. Deutsch and P. Jessen for stimulating discussions. We acknowledge financial support from IFRAF, ARDA/DTO and the European Integrated project SCALA. LCFIO is CNRS UMR8501. M.P.A.J. and A.M.L. are supported by Marie Curie Fellowships. A.G. is supported by a DGA Fellowship.

Correspondence and requests for materials should be addressed to A.B.

\section{Competing financial interests}

The authors declare no competing financial interests.

Reprints and permission information is available online at http://npg.nature.com/reprintsandpermissions/ 